\title{
Guest editorial: selected papers from Robotics: Science and Systems 2008
}

\author{
José Neira · Jeff Trinkle
}

Received: 3 March 2009 / Accepted: 10 March 2009 / Published online: 18 March 2009

(C) Springer Science+Business Media, LLC 2009

\section{Introduction}

This volume is one of two special journal issues compiled from the best papers presented at the fourth Robotics: Science and Systems (RSS) conference, held in Zurich in June 2008. As indicated by its title, RSS is a meeting that values research papers focusing on the development of, and experimentation with, new complex robotic systems in real-world environments as much as it values new theoretical and algorithmic advances. The second special issue will be published by the International Journal of Robotics Research.

The Zurich meeting of RSS received 163 submitted papers. After a rigorous review process, 40 papers were accepted covering a wide range of topics, from autonomous driving, to micro-robotics, to design of manipulators for use in functional magnetic resonance imaging, to grasping and manipulation. Following the tradition of RSS, there were a number of invited speakers who are global leaders in research areas on the frontiers of robotics. As an indication of the quality and inspirational value of the program, RSS Zurich was well attended, with nearly six times as many attendees as papers.

Papers selected for a special issue were selected first, and then partitioned into two groups for the two special issues. The papers selected for this special issue of Autonomous Robots have a common theme close to the focus of this journal: the study of problems related to mobile robots or

\section{J. Neira $(\bowtie)$}

Universidad de Zaragoza, Zaragoza, Spain

e-mail: jneira@unizar.es

J. Trinkle

Rensselaer Polytechnic Institute, Troy, USA

e-mail: trink@cs.rpi.edu vehicles immersed in dynamic environments that they must model in order to carry out their designated task. Another common trait in most papers is the use of machine learning techniques.

\section{Guide to the special issue}

The paper by Huang et al. presents the travel lane detector of Talos, the vehicle that won fourth place in the DARPA Urban challenge of 2007. This system is able to detect multiple painted travel lanes in urban road networks from image sequences and lidar data, using weak priors in the form of a topological road network, if available. No assumption is made about the position or orientation of the vehicle with respect to the road, allowing it to operate when changing lanes, at intersections, when exiting driveways and parking lots. This system successfully guided Talos through a $90 \mathrm{~km}$ course at speeds up to $40 \mathrm{~km} / \mathrm{h}$; the vehicle never unintentionally entered or exited a lane of travel. The authors present a detailed assessment of system performance.

The two following papers study the problem of detection and tracking of dynamic objects, in particular vehicles and pedestrians. The paper by Petrovskaya and Thrun describes the vehicle detection and tracking module of Junior, the robot that won second place in the Urban Grand Challenge in 2007. A high speed moving platform using laser range finders is used to detect and track moving vehicles. In contrast with typical vehicle tracking approaches that separate data segmentation and data association, in this system vehicles are modeled both dynamically and geometrically. Parameters of both models are estimated using a single Bayes filter. The system also provides means to detect poorly visible black vehicles from the laser scans, and is robust to the high signal-to-noise ratio that results from using relatively low 
speed sensors to detect fast moving vehicles. Experimental validation includes data from challenging traffic situations in the Urban Grand Challenge and other urban settings.

The paper by Luber $e t$ al. presents an unsupervised learning approach for detecting, tracking and classifying dynamic objects in sequences of planar laser range scans. An exemplar-based model is used to represent the time-varying appearance of moving objects; the sensor stream is preprocessed using feature detector and tracking algorithms, and an unsupervised clustering scheme automatically builds appropriate class assignments. The system is able to learn and reuse object models on-the-fly without any human intervention. Both supervised and unsupervised learning experiments are carried out using a SICK laser range scanner, in a scenario that includes walking and running pedestrians, cyclists at different speeds, people pushing a buggy or pulling a suitcase or on kangaroo shoes. Results show that the system can discriminate accurately between object classes, given the limited information provided by the laser data and the high level of self-occlusion.

The next two papers deal with the problem of modeling the environment traversed by a mobile robot. The paper by Posner et al. proposes a multi-level classifier for semantic annotation of urban maps. Environment data is obtained by an onboard color camera and a 3D laser scanner. The twostage classifier considers local scene properties (using a bagof-words classifier), and spatial and temporal context (via a Markov Random Field). An information-theoretic bail-out test helps identify and exclude unlikely exemplars, resulting in important computational savings. Scene labeling including classes such as pavement, grass, smooth or textured walls, foliage and vehicle, is attained at speeds suitable for online execution. The system performance is analyzed over a $17 \mathrm{~km}$ trajectory through Oxford.

The paper by Guigère and Dudek describes an automatic terrain classifier, an off-line windowless clustering algorithm that exploits temporal dependency between consecutive measurements. The system is capable of clustering data that have either high process or high observation noise. It also works well in situations in which the system switches states too frequently to find a suitable probability density function to describe the distribution or to detect changes. This avoids the overestimation of the transition likelihood, common to other algorithms. The algorithm computes sets of parameter values that minimize a cost function related to change in classifier probability outputs over time. This system is tested on both synthetic and real data sets, a hexapod robot over several indoor and outdoor surfaces using internal sensors for data collection, and a differential drive robot over indoor surfaces using an external tactile sensor for data collection.

The last paper of the Special Issue, by Stachniss et al. studies a problem of practical importance, learning two dimensional models of gas distributions using mobile robots, a challenging problem due to the chaotic nature of gas dispersal and the high sparsity of the available data. A sparse Gaussian mixture model is used to represent both the smooth background signal and high gas concentration areas, an attractive modeling technique that provides both gas concentration estimates and the predictive uncertainty. A generic algorithm to learn a Gaussian Process mixture model is proposed to reduce model complexity and achieve efficient representations for large data sets. Experiments are carried out with a mobile robot equipped with an electronic nose, showing superior predictive ability, as compared with previous methods.

We hope you enjoy these papers as much as we did!

Acknowledgements The Guest Editors wish to express their gratitude to Gaurav Sukhatme, the AUTONOMOUS ROBOTS Editor-inChief, as well as Senior Editor Melissa Fearon, Editorial Assistant Valerie Schofield, Journal Manager Gary Anderton, and JEO assistant Rolan Profugo, for their constant support. They are also grateful to the members of the Organizing Committee of RSS 2008, who first managed the papers that appear in this Special Issue. They are finally enormously grateful to the many colleagues who agreed to provide reviews for prior and final versions of the papers selected for Special Issue.

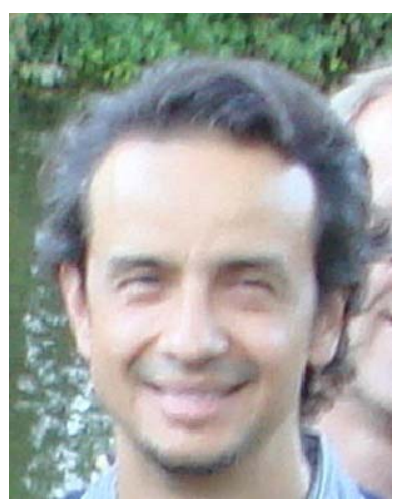

José Neira was born in Bogota, Colombia, in 1963. He received the M.S. degree in computer science from the Universidad de los Andes, Colombia, in 1986, and the Ph.D. degree in computer science from the University of Zaragoza, Spain, in 1993. He is Associate Professor in the Departamento de Informatica e Ingenieria de Sistemas, University of Zaragoza, where he is in charge of courses in compiler theory, computer vision and mobile robotics. His current research interests include autonomous robotics and environment modelling. José has been guest editor of the IEEE Transactions on Robotics special issue on Visual SLAM, together with Andrew Davison, Imperial College London, and John Leonard, MIT. He is currently associate editor of the IEEE Transactions on Robotics, and guest editor of Robotics and Autonomous Systems for the special issue Inside Data Association, together with Udo Frese, University of Bremen. He is also area chair for the Robotics: Science and Systems 2009 conference, and local arrangements chair for Robotics: Science and Systems 2010. 


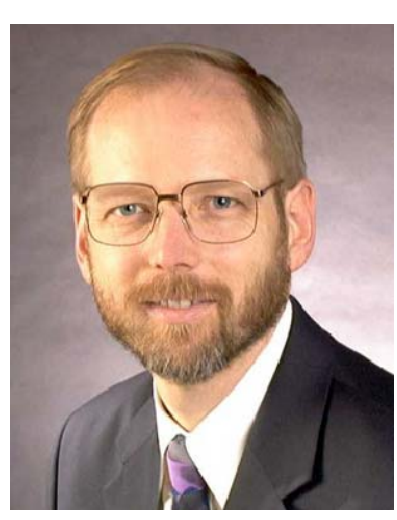

Jeff Trinkle received his bachelor's degrees in Physics (1979) and Engineering Science and Mechanics (1979) from Ursinus College and Georgia Institute of Technology, respectively. In 1987, he received his $\mathrm{PhD}$ from the Department of Systems Engineering at the University of Pennsylvania, where he was a research assistant in the GRASP Laboratory. Since 1987, he has held faculty positions in the Department of Systems and Industrial Engineering at the University of Arizona and the Department of Computer Science at
Texas A\&M University. From 1998 to 2003 he was a research scientist at Sandia National Laboratories in Albuquerque New Mexico. He is now Professor of Computer Science at Rensselear Polytechnic Institute in Troy, New York, where he served as Department Chair from 2003 to 2009. His primary research interests are in the areas of robotic manipulation, multibody dynamics, and automated manufacturing. 